\title{
Osteoclasts May Affect Glucose Uptake-Related Insulin Resistance by Secreting Resistin
}

\author{
Xiangqi Li* \\ Fei Sun* \\ Jiancan Lu \\ Jichen Zhang \\ Jingnan Wang \\ Hongling Zhu \\ Mingjun Gu \\ Junhua Ma
}

Department of Endocrinology, Shanghai Gongli Hospital, The Second Military

Medical University, Shanghai, 200I35,

People's Republic of China

*These authors contributed equally to this work
Correspondence: Junhua $\mathrm{Ma}$

Department of Endocrinology, Shanghai Gongli Hospital, The Second Military

Medical University, Miaopu Road 219 ,

Shanghai, 200135, People's Republic of China

Tel +86 2I 58858730

Email jhmag|@|26.com
Objectives: Bone may play a role in the modulation of insulin sensitivity. Insulin resistance can be caused by increased resistin. However, whether osteoclasts affect the insulin resistance via resistin remains unclear. In the present study, we show the expression of resistin in osteoclasts and the possible underlying role of resistin on glucose uptake-related insulin resistance in vitro. Methods: Conditioned mediums (CM) were collected from Raw264.7 cells treated without (CCM) or with RANKL (CM3, treated with RANKL for 3 days; CM5, treated with RANKL for 5 days) and transfected with control or resistin siRNA (CMsiRNA). The osteoclast formation was examined by tartrate resistant acid phosphatase (TRAP) staining. C2C12 myoblasts were cultured with the CM or CMsiRNA. Glucose uptake was evaluated by 2-NBDG fluorescence intensity. Resistin expression was evaluated by quantitative realtime polymerase chain reaction (qRT-PCR) and enzyme-linked immunosorbent assay. Statistical analysis was performed by an independent two sample $t$-test or one-way ANOVA. Results: The 2-NBDG fluorescence intensity was higher in $\mathrm{C} 2 \mathrm{C} 12$ cells treated with $\mathrm{CCM}$ compared to those that received CM3 and CM5 $(\mathrm{p}<0.05)$. Resistin mRNA and protein expressions were both increased in RAW264.7 cells treated with RANKL for 3 days and 5 days compared with those cells without RANKL administration. The 2-NBDG fluorescence intensities in $\mathrm{C} 2 \mathrm{C} 12$ cells treated with CMsiRNA and CM5+Anti-resistin antibody were significantly higher than those cultured with CM5 ( $\mathrm{p}<0.05)$.

Conclusion: Osteoclasts may promote glucose uptake-related insulin resistance by secreting resistin.

Keywords: bone, insulin resistance, osteoclast, resisin, diabetes mellitus

\section{Introduction}

Insulin resistance is a pathophysiological condition in which cells in muscles, fat, and liver do not respond well to normal doses of insulin and cannot use glucose from the blood for energy. ${ }^{1}$ Insulin resistance is one of the critical reasons for the pathogenesis of multiple metabolic syndrome, including type 2 diabetes mellitus and obesity. ${ }^{2}$

Recently, an increasing number of studies have shown that bone is one type of endocrine organ which can regulate the energy metabolism. ${ }^{3,4}$ Obesity, high blood sugar levels and insulin resistance occurred in osteocalcin (OC) knockout mice. ${ }^{3}$ Insulin can promote osteoblasts to produce $\mathrm{OC}$, and the bone regulates carbohydrate metabolism via OC. Osteoclast is another type of cell in bone. However, the role of osteoclast on insulin resistance has not been totally clarified. A recent study indicated that denosumab, a monoclonal antibody that binds with high specificity to the human receptor activator of NF-kB ligand (RANKL), could significantly diminish the insulin resistance condition in the fourth week. ${ }^{5}$ Similar trends were observed in the 12 th and 
24th week, but without statistical significance. Interestingly, Passeri and colleagues showed that denosumab might positively affect hepatic insulin sensitivity. ${ }^{6}$ The in vivo study indicated that blockage of RANKL signaling in mouse models resulted in a marked improvement of hepatic insulin sensitivity. ${ }^{7}$ We speculate that osteoclast may play an important role in insulin resistance because RANKL is a critical cytokine for osteoclast formation. However, whether osteoclasts affect the insulin resistance and the possible underlying mechanisms are not completely understood.

Resistin is a type of adipocytokines which is produced by adipocytes. ${ }^{8}$ It acts on myocytes, hepatocytes, and adipocytes, reducing their sensitivity to insulin. ${ }^{9}$ It also takes part in the inflammatory response and increases the expression of some proinflammatory cytokines. ${ }^{10}$ Insulin resistance can be caused by resistin-related chronic inflammation. Resistin can induce insulin resistance by both AMPK-dependent and AMPK-independent pathways. $^{11}$ It has been shown that resistin is expressed in human macrophages, placenta, and pancreas. ${ }^{12-}$

${ }^{14}$ Inflammatory cells such as macrophages are considered the predominant source of circulating resistin. ${ }^{13}$ A recent study showed that resistin independently and positively correlated with fasting glucose in elderly people with metabolic syndrome. ${ }^{15}$ Preosteoclasts and osteoclasts are both macrophages. Thommesen et $\mathrm{al}^{16}$ showed that the expression of resistin mRNA in RAW 264.7, a type of preosteoclastic cell, was increased during differentiation. Recently, resistin expression was also up-regulated in periodontal cells and tissues that had periodontitis. ${ }^{17}$ However, whether osteoclasts affect the insulin resistance via resistin remains unclear. Insulin resistance was defined as a reduced sensitivity to insulin by the body's insulin-dependent processes, such as glucose uptake. ${ }^{1}$ In the present study, we aimed to show the effect of osteoclasts on insulin resistance from the aspect of glucose uptake and the possible underlying role of resistin.

\section{Materials and Methods Cell Culture}

RAW264.7 cells and C2C12 cells were obtained from National Collection of Authenticated Cell Cultures (Shanghai, China). RAW264.7 cells were cultured in Dulbecco's modified Eagle's Medium (DMEM; 25 mM glucose; Gibco, USA) containing $10 \%$ FBS, $1 \%$ penicillin/streptomycin, and with $20 \mathrm{ng} / \mathrm{mL}$ RANKL at $37{ }^{\circ} \mathrm{C}$ in a humidified atmosphere of $95 \%$ air and $5 \% \mathrm{CO}_{2}$. On the third or fifth day, the cells were cultured with RANKL-free and glucose-free medium for $12 \mathrm{~h}$. Then, the medium was collected for indirect co-culturing with $\mathrm{C} 2 \mathrm{C} 12$ cells. The medium from RAW264.7 cells without RANKL induction was also collected as control.

C2C12 myoblasts were cultured in DMEM supplemented with 10\% FBS (Gibco, USA), $100 \mathrm{U} / \mathrm{mL}$ penicillin, and $100 \mu \mathrm{g} / \mathrm{mL}$ streptomycin at $37^{\circ} \mathrm{C}$ in a humidified atmosphere of $95 \%$ air and 5\% CO2. On the second day, the medium was changed to DMEM with $2 \%$ horse serum (Gibco, USA) to induce differentiation for an additional 4 days. The medium was replaced every two days.

The differentiated $\mathrm{C} 2 \mathrm{C} 12$ myoblasts $\left(10^{4}\right.$ cells/well $)$ were cultured with the conditioned mediums (CMs) containing 40\% medium collected from RAW264.7 cells (non-RANKL-treated, CCM; RANKL-treated for 3 days and 5 days, CM3 and CM5) and $60 \mathrm{mM}$ of glucose $(\mathrm{n}=6)$ for $24 \mathrm{~h}$ in a 24 -well plate.

\section{RNA Interference}

Resistin siRNA (sense 5'-CCAAAUGCAAUAAAGAAC AUUGGCU-3'; antisense 5'-AGCCAAUGUUCUUU AUUGCAUUUGG-3'; each strand with a 3' dTdT overhang) and negative control (NC) siRNA (sense 5'TTCTCCGAACGTGTCGACACGGAGAC -3'; antisense 5'-TAATGTACGTGCAGTTAGCCTGACGC -3') were obtained from Life Technologies. Transfection of siRNA was performed using Lipofectamine RNAiMAX and Opti-MEM (both from Life Technologies) according to the manufacturer's instructions. Raw264.7 cells were cultured as previously described. On day 2, $50 \mathrm{nM}$ of each siRNA was added in a maturation medium and cultured. On the third or fifth day, the cells were cultured with RANKL-free and glucose-free medium for $12 \mathrm{~h}$. Then, the medium (NC, CCMi, CM3i and CM5i) was collected for the indirect co-culturing with $\mathrm{C} 2 \mathrm{C} 12$ cells.

\section{Cell Viability}

The cell viability of RAW264.7 cells transfected with NC and resistin siRNA was evaluated by a CCK8 kit. Briefly, RAW264.7 cells transfected with $\mathrm{NC}$ and resistin siRNA were seeded into 96-well plates for $24 \mathrm{~h}$. Then, $10 \mu \mathrm{L}$ of CCK8 solution was added to each well and the plate was incubated in the incubator for 4 hours. The absorbance of the cells was measured at $450 \mathrm{~nm}$ using a microplate reader. Similarly, the effects of $\mathrm{CMs}$ on $\mathrm{C} 2 \mathrm{C} 12$ viability were also evaluated by the CCK8 kit.

\section{Glucose Uptake Assay}

C2C12 cells treated with the CMs were washed with PBS and starved in glucose-free DMEM containing 2\% FA-free 
FBS for $3 \mathrm{~h}$. They were subsequently incubated in the presence of insulin $(100 \mathrm{nM})$ for $10 \mathrm{~min}$, then washed with ice-cold Krebs buffer and 2-NBDG (Cayman, USA) $(150 \mu \mathrm{g} / \mathrm{mL})$ was added into the culture medium for 30 $\min$ at $37{ }^{\circ} \mathrm{C}$. The 2-NBDG fluorescence intensity was measured using a microplate reader at an excitation wavelength of $480 \mathrm{~nm}$ and an emission wavelength of $540 \mathrm{~nm}$.

In addition, $10 \mathrm{ng}$ anti-resistin antibody (ab136877, Abcam, USA) was added into the obtained $20 \mathrm{~mL}$ CM5 (CM5-AntiR). Then, the $\mathrm{C} 2 \mathrm{C} 12$ cells were cultured with the mixed medium. Glucose uptake was assessed using the 2-NBDG fluorescence intensity. The CMM value for the glucose uptake was set as $100 \%$.

\section{Osteoclast Formation}

RAW264.7 cells could differentiate to osteoclasts in the presence of receptor activator of nuclear factor- $\kappa$ B ligand (RANKL) induction. The osteoclast formation was evaluated by tartrate resistant acid phosphatase (TRAP) staining. Briefly, RAW264.7 cells were washed with cold PBS, fixed with $4 \%$ paraformaldehyde for $10 \mathrm{~min}$, and stained for TRAP using a commercial kit (Sigma-Aldrich, USA). The TRAP-positive multinucleated cells containing three or more nuclei were counted as mature osteoclasts. The resistin mRNA expression during osteoclast formation ( 0,3 and 5 days) was observed $(n=4)$.
Primary osteoclasts were also isolated from new-born Sprague-Dawley rats. The study protocol was approved by Animal Care Committee of Shanghai Gongli Hospital. All experiments were conducted according to Guiding Principles for Care and Use of Animals (National Research Council, 8th edition). Briefly, the long bones were placed in alpha-MEM and the soft tissues were removed. Then, the bones were curetted and the dispersed cell solution was seeded into a 6-well plate for $30 \mathrm{~min}$. The osteoclasts were attached to the plate. The cells were used for RNA extraction and supernatants were used for the resistin protein assay at $24 \mathrm{~h}$ and $72 \mathrm{~h}(\mathrm{n}=3)$.

\section{RNA Extraction and Quantitation of mRNA Expression by Quantitative Real-Time Polymerase Chain Reaction (qRT-PCR)}

Total RNA was isolated from osteoclasts by using Trizol reagent. Complementary DNA was synthesized by using a Quantscript RT kit (Tiangen Biotech Co., China) (0.5 $\mu \mathrm{g}$ total RNA). The individual cDNA species were amplified in a reaction mixture containing cDNA aliquot (50 ng), and the relevant sense and antisense primers (Resistin: forward 5'TCACTTTTCACCTCTGTGGATATGAT-'3, reverse 5'TGCCCCAGGTGGTGTAAA-'3). Then, real-time PCR was performed using an ABI PRISM 7500 Sequence Detection
A

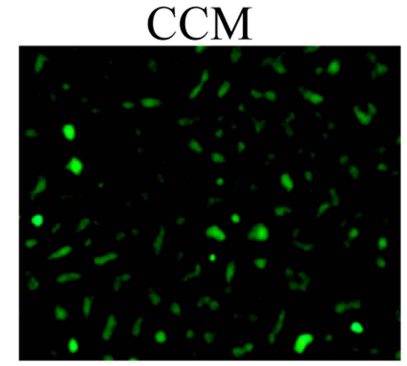

B

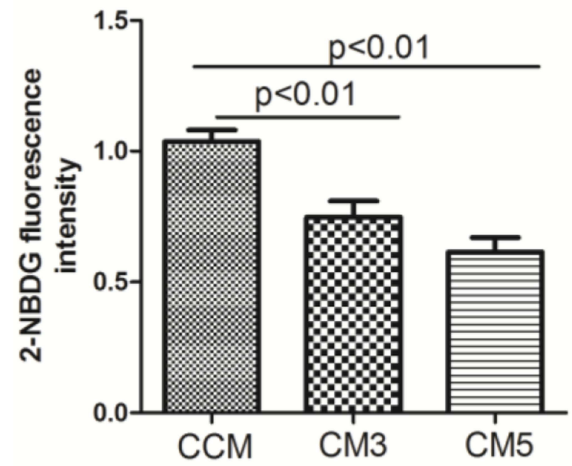

CM3

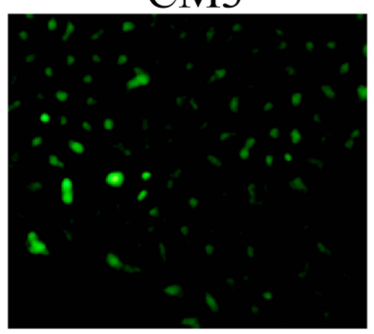

C

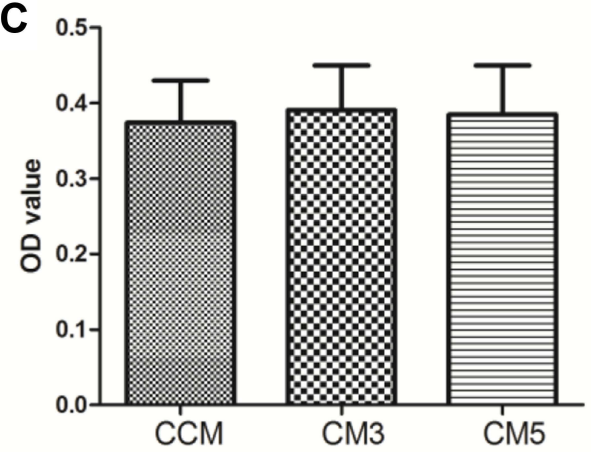

Figure I Osteoclast affected glucose uptake in $\mathrm{C} 2 \mathrm{Cl} 2$ cell (A and $\mathbf{B}$ ) and effects of conditioned mediums (CMs) on cell viability (C). The 2-NBDG fluorescence intensity were significantly decreased in CM3 and CM5 groups. No significant differences were observed in cell viability cultured with CMs. CCM: conditioned medium from RAW264.7 without RANKL induced; CM3: conditioned medium from RAW264.7 treated with RANKL for 3 days; CM5: conditioned medium from RAW264.7 treated with RANKL for 5 days. 
System (Life Technologies) and SYBR-Green. $\beta$-actin mRNA (forward 5'-TTCCTTCTTGGGTATGGAAT-'3, reverse 5'GAGCAATGATCTTGATCTTC-' 3 ) was used as an internal control. We also detected leptin (forward 5'GGATCAGGTTTTGTGGTGCT-'3, reverse 5'-TTGTGGC CCATAAAGTCCTC-' 3 ) and adiponectin mRNA (forward 5'-CAGGTCTTCTTGGTCCTAAGGG-' 3 , reverse 5'GTCCACATTCTTTTCCTGATACTG-'3) expression. The reactions protocol was as follows: initiated at $94{ }^{\circ} \mathrm{C}$ for $5 \mathrm{~min}$ and PCR $\left(94{ }^{\circ} \mathrm{C}\right.$ for $5 \mathrm{~s}, 60^{\circ} \mathrm{C}$ for $\left.20 \mathrm{~s}\right)$ for 35 cycles. Relative mRNA expression was determined using the $2-{ }^{\Delta \Delta} \mathrm{Ct}$ method.

\section{Enzyme Linked Immunosorbent Assay (ELISA)}

Resistin released from RAW264.7 cells was determined using the Mouse Resistin Quantikine ELISA
Kit (R\&D Systems, Minneapolis, MN, USA) according to the manufacturer's instructions. The detection limit was $8 \mathrm{pg} / \mathrm{mL}$. The $\mathrm{CV}$ of the intra-assay and interassay was lower than $7 \%$. The recovery rate was $85-108 \%$.

\section{Statistical Analysis}

All statistical analyses were performed by using SPSS 20 (IBM Corp., Armonk, NY, USA). Normal distribution was checked by Shapiro-Wilk test. The data are shown as mean $\pm \mathrm{SD}$ (normal distribution) or median (interquartile range). Statistical analysis was performed by an independent two sample $t$-test or One-way ANOVA. $\mathrm{p}<0.05$ was considered to be statistically significant.
A
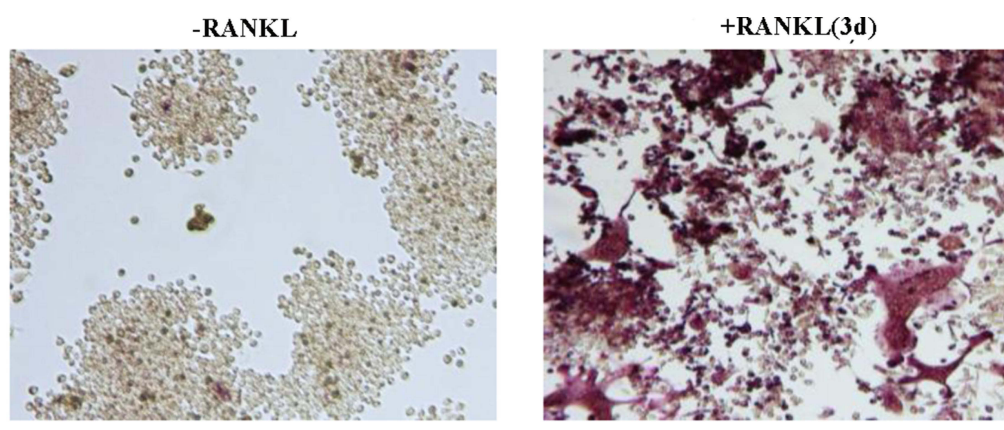

B

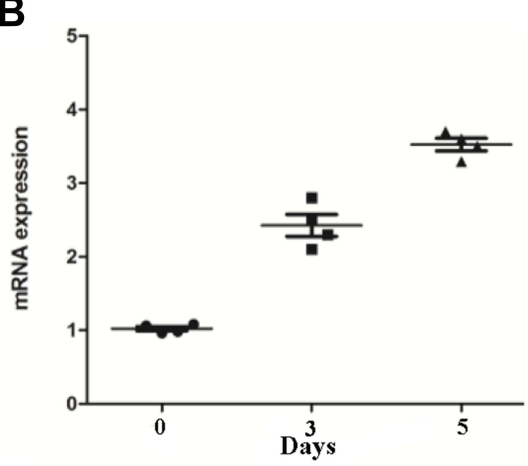

E

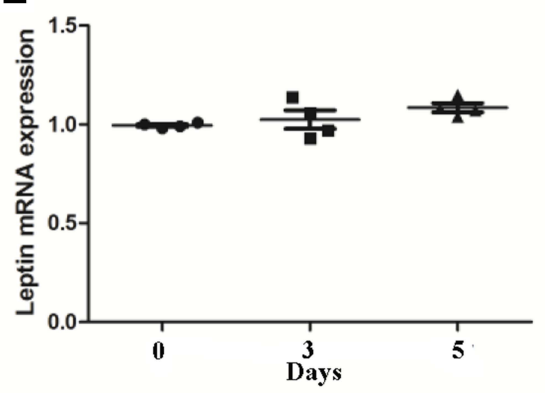

C
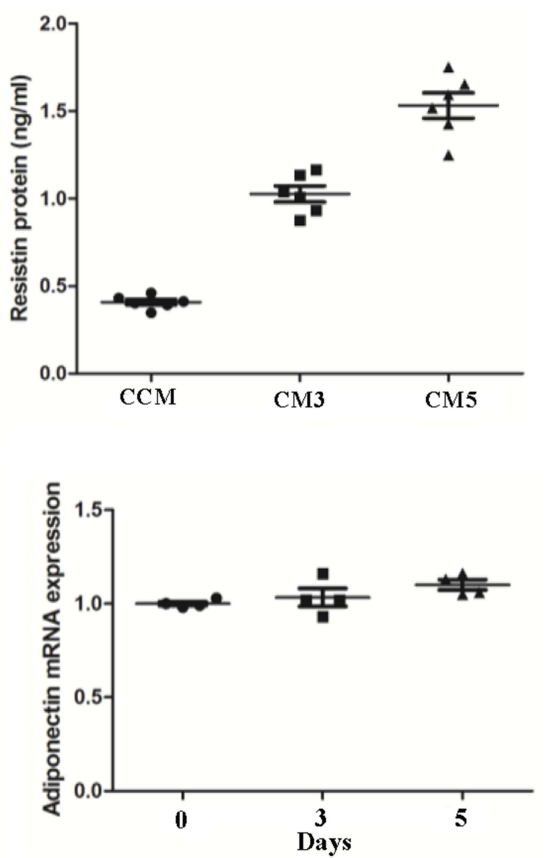

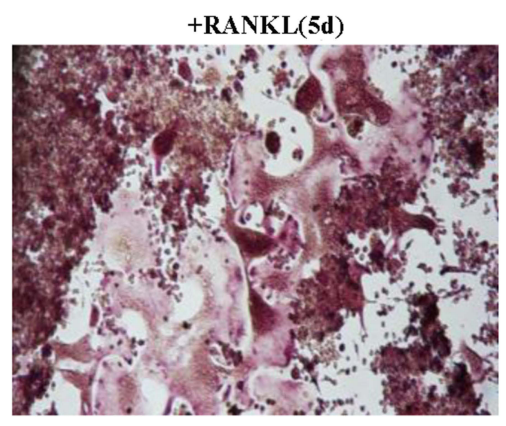

D

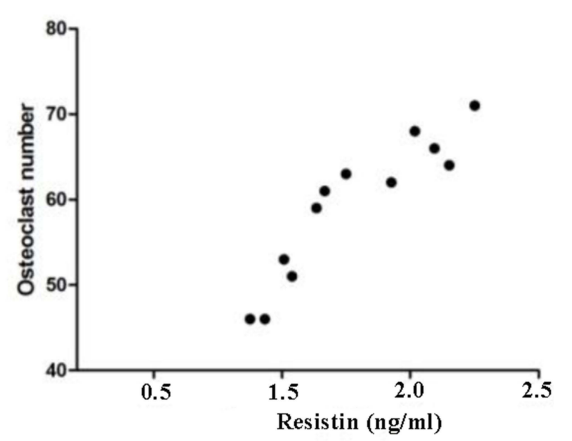

Figure 2 Resistin expression during osteoclast formation. RANKL promoted RAW264.7 cell differentiation to osteoclast (A). The relative mRNA (B) and protein (C) expression were both increased in RANKL treated cells $(n=3)$. The resistin protein level was positively correlated with osteoclast number $(D)$. There were no significant changes in leptin and adiponectin mRNA during osteoclast formation (E). CCM: conditioned medium from RAW264.7 without RANKL induced; CM3: conditioned medium from RAW264.7 treated with RANKL for 3 days; CM5: conditioned medium from RAW264.7 treated with RANKL for 5 days. 


\section{Results}

\section{Osteoclasts Affected Glucose Uptake in $\mathrm{C} 2 \mathrm{Cl} 2$ Cells}

Firstly, we determined the glucose uptake in $\mathrm{C} 2 \mathrm{C} 12$ cells treated with different CMs. The glucose uptake was decreased in $\mathrm{C} 2 \mathrm{C} 12$ cells treated with CM3 and CM5 compared to those that received CCM $(p<0.01)$. As shown in Figure 1, the 2-NBDG fluorescence intensity was significantly decreased in the CM3 and CM5 groups (Figure 1A and $\mathrm{B}$ ). There were no significant differences in cell viability in $\mathrm{C} 2 \mathrm{C} 12$ cells treated with CMs (Figure 1C).

\section{Resistin Expression During Osteoclast Formation}

Subsequently, we determined the mRNA and protein expression of resistin during osteoclast formation (Figure 2). RAW264.7 differentiated into osteoclasts in the presence of RANKL induction (Figure 2A). Relative resistin mRNA (Figure 2B) and protein expressions (Figure 2C) were both increased in RAW264.7 cells treated with RANKL for 3 days and 5 days compared with those cells without RANKL administration. The resistin level was positively correlated with the osteoclast number $(\mathrm{r}=0.63, \mathrm{p}<0.05)$ (Figure 2D). However, no significant differences were observed in leptin and adiponectin mRNA during during osteoclast formation.
The number of osteoclasts at $72 \mathrm{~h}$ was increased compared to that at $24 \mathrm{~h}$ (Figure 3A). The primary osteoclast model also showed that relative resistin mRNA (Figure 3B) and protein expressions (Figure 3C) were also increased with the increase in osteoclast formation.

\section{Resistin Knockdown}

The fluorescent images showed that resistin siRNA and negative control siRNA were transfected into the RAW264.7 cells (Figure 4A). The transfection did not show any obvious cytotoxic effects (Figure 4B). The relative resistin mRNA and protein expression in cells treated with resistin siRNA were significantly lower than those treated with negative control siRNA (Figure 4C and D). Relative resistin mRNA expression was decreased by approximately $65 \%$ of that of the negative control siRNA-transfected cells (Figure 4E). ELISA further showed that the resistin protein was also decreased in cells transfected with resistin siRNA (Figure 4F).

\section{Glucose Uptake with Resistin Knockdown}

The $\mathrm{C} 2 \mathrm{C} 12$ cells were cultured with the CCMi and CM5i collected from the RANKL-induced RAW264.7 cells transfected with resistin siRNA. The 2-NBDG fluorescence intensity is shown in Figure 5. The intensity was significantly increased in $\mathrm{C} 2 \mathrm{C} 12$ cells cultured with $\mathrm{CM} 5 \mathrm{i}$ compared with the CM5 group ( $\mathrm{p}<0.05)$.
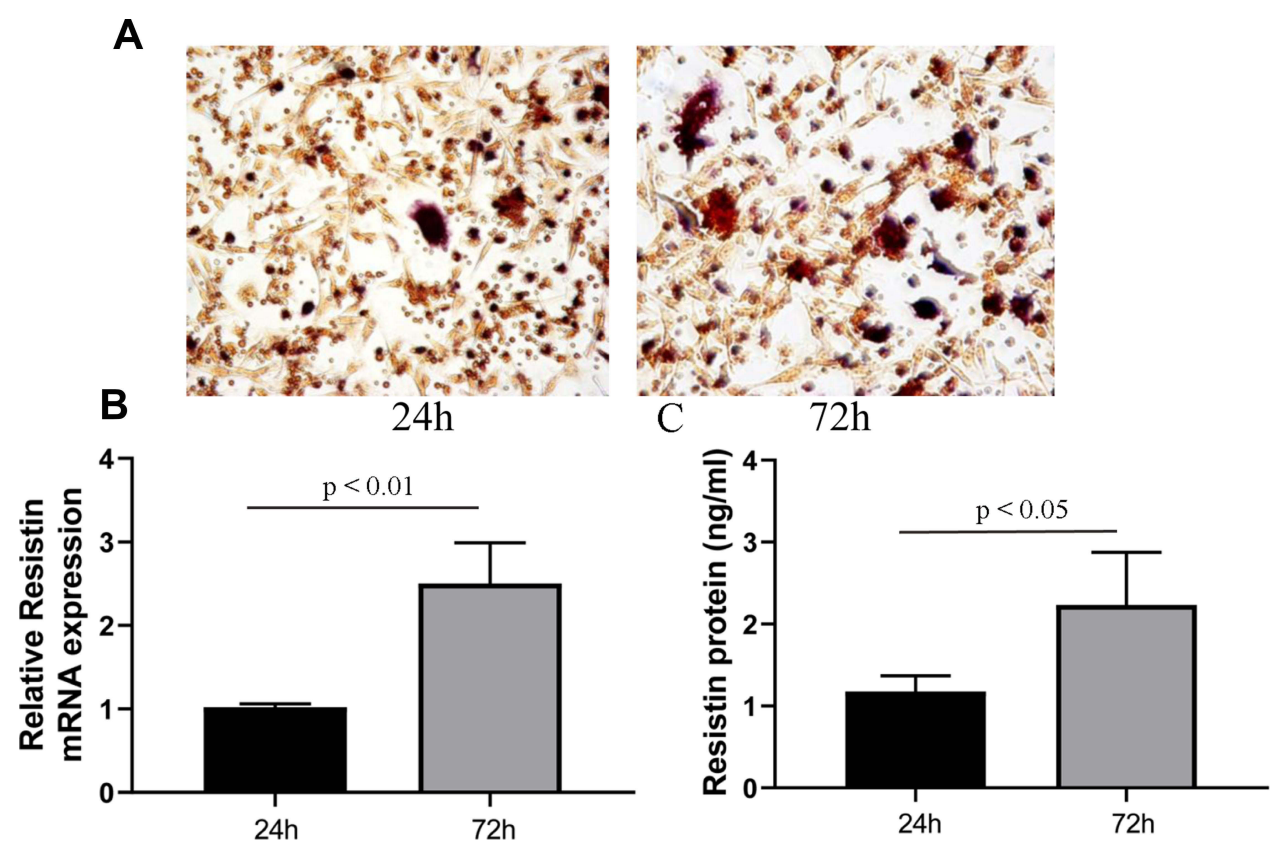

Figure 3 Resistin expression in primary osteoclast. Primary osteoclast was isolated from the long bone of new-born rat. The number of osteoclast at $72 \mathrm{~h}$ was larger than that at $24 \mathrm{~h}(\mathbf{A})$. The relative mRNA (B) and protein $(\mathbf{C})$ expression at $72 \mathrm{~h}$ were also higher than those at $24 \mathrm{~h}(\mathrm{n}=3)$. 


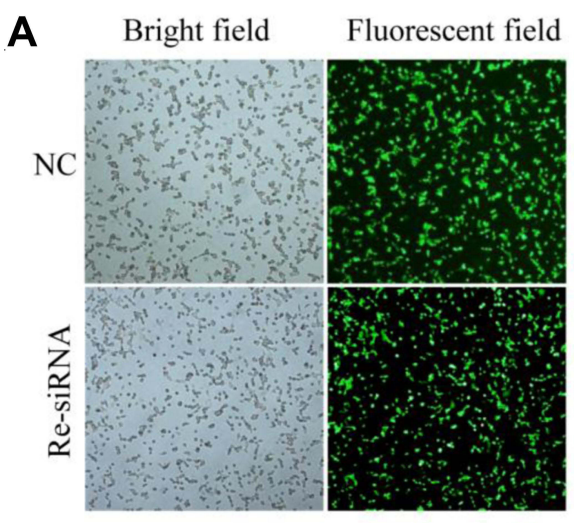

C

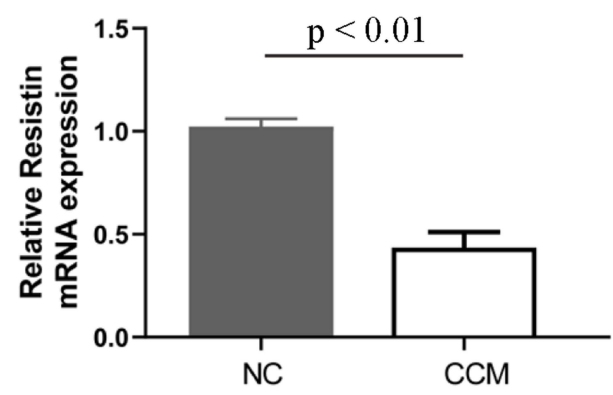

E

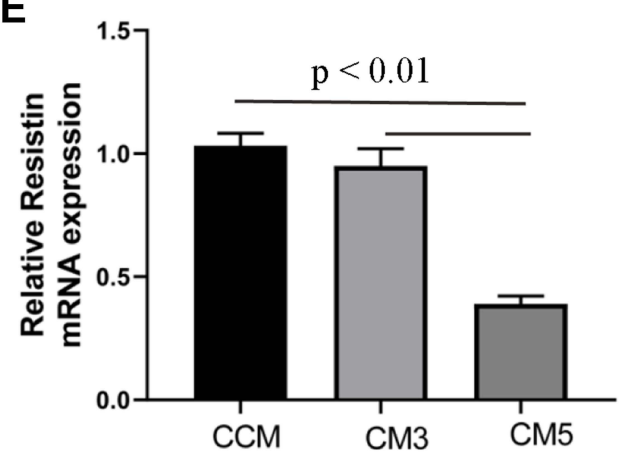

B
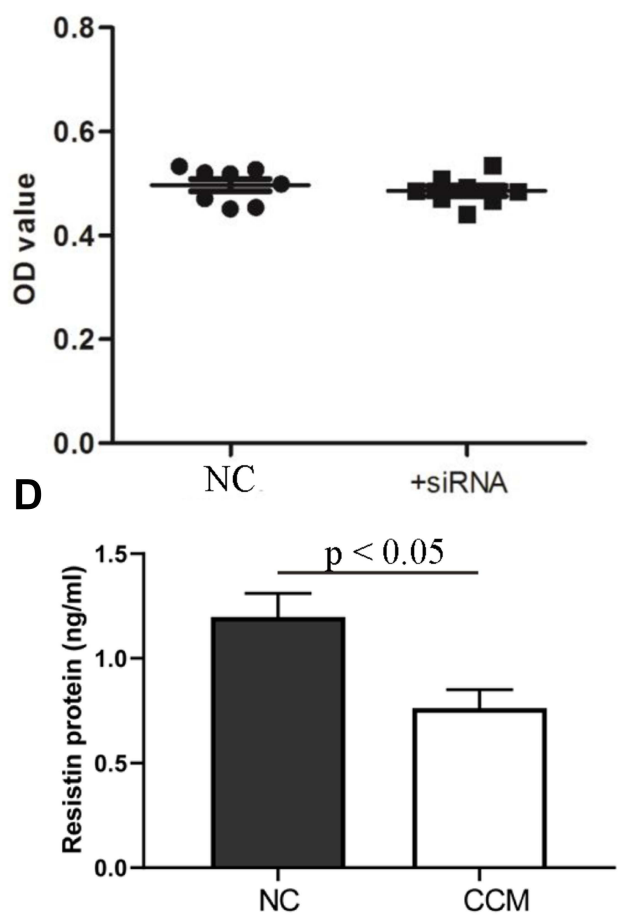

$\mathbf{F}$

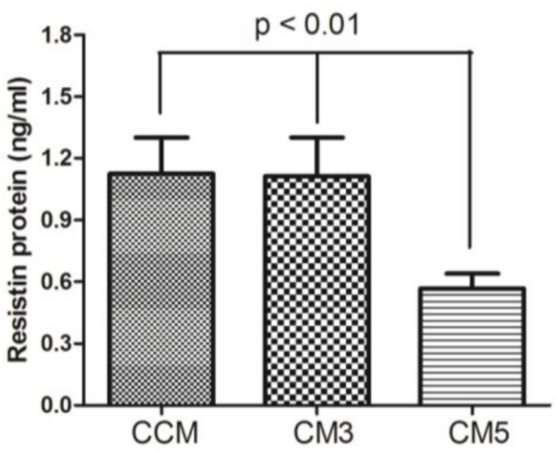

Figure 4 Resistin knockdown by siRNA. Resistin siRNA and negative control (NC) siRNA were transfected into the RAW264.7 cells (A). The transfection did not show obviously cytotoxic effects $(\mathbf{B})(n=8)$. Relative resistin mRNA and protein expression were decreased in resistin siRNA $(C C M)$ compared NC group $(\mathbf{C}$ and $\mathbf{D})$ ( $n=4)$. In addition, relative resistin mRNA $(\mathbf{C})$ and protein $(\mathbf{D})$ expression at 3 days and 5 days of RANKL induction were also observed $(\mathbf{E}$ and $\mathbf{F})(\mathrm{n}=4)$. NC: negative control siRNA; CCMi: conditioned medium from RAW264.7 transfected with resistin siRNA, but without RANKL induced; CM3i: conditioned medium from RAW264.7 transfected with resistin siRNA and treated with RANKL for 3 days; CM5i: conditioned medium from RAW264.7 transfected with resistin siRNA and treated with RANKL for 5 days.

\section{Glucose Uptake in $\mathrm{C} 2 \mathrm{Cl} 2$ Cells Cultured with CM5-AntiR}

The 2-NBDG fluorescence intensity is shown in Figure 6. The intensity was significantly increased in $\mathrm{C} 2 \mathrm{C} 12$ cells cultured with CM5-AntiR compared with the CM5 group $(p<0.05)$.

\section{Discussion}

Bone cells not only control bone remodeling, but also modulate adipose sensitivity and insulin secretion. ${ }^{18}$ Adipocytokines, such as adiponectin, resistin and vaspin, were suspected to participate in metabolic disorders and insulin resistance. ${ }^{19,20}$ Thommesen et $\mathrm{al}^{16}$ showed that the expression of resistin mRNA in preosteoclastic cells. However, few studies have shown the association between osteoclast differentiation and insulin resistance. Our data demonstrated that osteoclast differentiation may induce insulin resistance by secreting resistin. These results were further confirmed in a resistin knockdown cell model.

The association between osteoblasts and glucose homeostasis has been reported. ${ }^{18}$ Osteocalcin produced by the osteoblasts has been shown to affect adipocyte sensitivity to insulin and insulin secretion. ${ }^{3,18}$ However, few studies have investigated the effects of osteoclasts on glucose homeostasis or insulin resistance. Epidemiological data 
$\mathrm{CCM}$
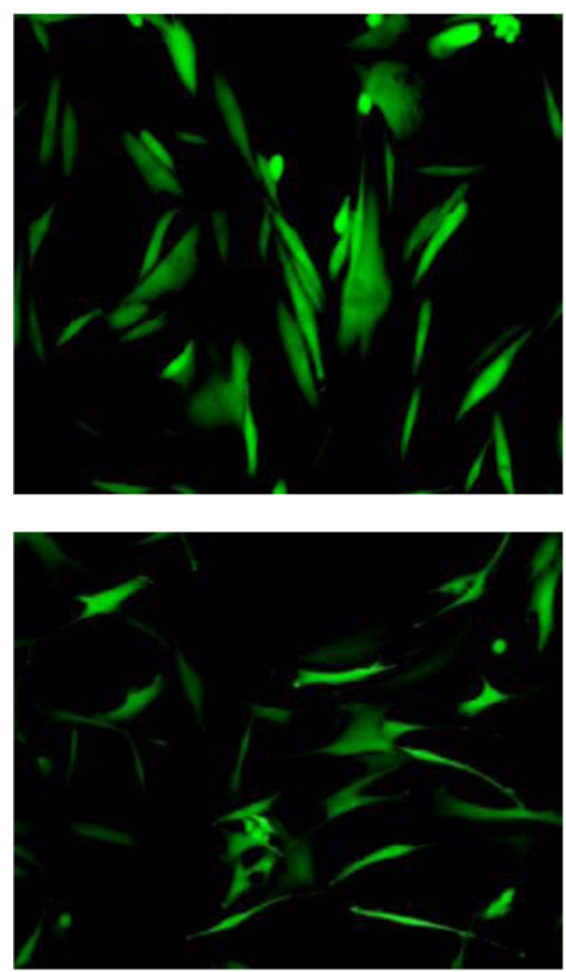

CMsiRNA
CM5
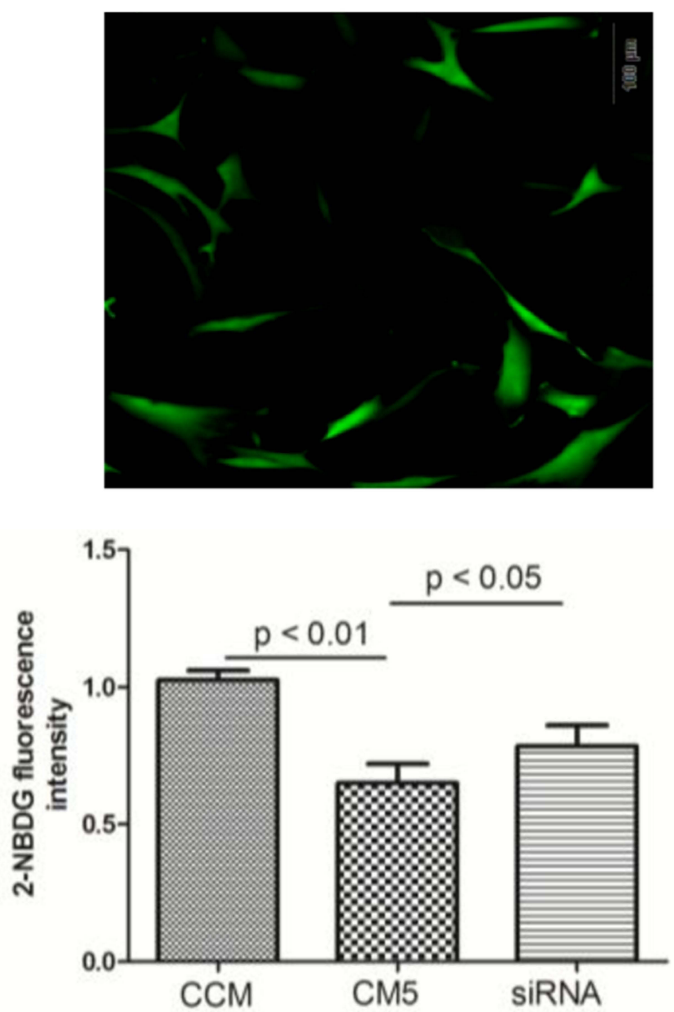

Figure 5 Glucose uptake in $\mathrm{C} 2 \mathrm{Cl} 2$ cell treatment with different conditioned medium (CCM, CM5 and CMsiRNA). The 2-NBDG fluorescence intensity was significantly increased in $\mathrm{C} 2 \mathrm{Cl} 2$ cells cultured with CMsiRNA compared with CM5 group $(\mathrm{p}<0.05)(n=4)$. CCM: conditioned medium from RAW264.7 without RANKL induced; CMsiRNA: conditioned medium from RAW264.7 transfected with resistin siRNA and treated with RANKL for 5 days; CM5: conditioned medium from RAW264.7 treated with RANKL for 5 days.

indicated that the concentration of soluble RANKL, a key cytokine in osteoclast differentiation, is an independent risk factor of type 2 diabetes mellitus. ${ }^{7}$ Interestingly, several reports show that inhibition of RANKL may improve insulin resistance in people and animal model. ${ }^{5,7}$ These studies indicated that osteoclast differentiation may contribute to insulin resistance. Our data evidenced that this hypothesis may be correct. The conditioned medium obtained during osteoclast differentiation induced glucose uptake-related insulin resistance in $\mathrm{C} 2 \mathrm{C} 12$ cells.

Few data were found regarding the mechanism by which RANKL affected the glucose homeostasis. Kiechl et $\mathrm{al}^{7}$ showed that RANKL can affect the hepatic insulin resistance. They developed a hepatocyte-specific Rank (which could bind with RANKL) knockout ( Rank $^{\mathrm{ko}}$ ) mice and compared them with wild-type controls. The control mice developed insulin resistance after 4 weeks of a high-fat diet, but Rank ${ }^{\mathrm{ko}}$ did not. Similar results were observed in mice that received a hydrodynamic injection of Rank shRNA (RANKi). They also found that specific deletion of Rank in the skeletal muscle or in pancreatic $\beta$-cells did not show improved insulin sensitivity. Therefore, they speculated that the role of RANKL on insulin resistance was due to its effects on hepatocytes. Osteoclasts are also target cells to RANKL. RANK is also expressed in preosteoclasts and mature osteoclasts. However, the role of osteoclasts in RANKL-related insulin resistance was not investigated in their study. Interestingly, preosteoclastic cells can express resistin, ${ }^{16}$ which is associated with metabolic impairments and insulin resistance. ${ }^{21,22}$ Our data showed that the resistin levels were increased during osteoclast differentiation. When the expression of resistin was downregulated by Resistin siRNA, C2C12 cells did not show abnormal glucose uptake. Our results indicated that osteoclasts may affect glucose uptake by resistin.

Many population and animal studies have shown positive correlation between resistin level and fasting blood glucose. ${ }^{15,23}$ Resistin may be involved in the regulation of glucose metabolism. However, no significant relationship between plasma levels of resistin and glucose metabolism was also reported in a number of population studies. ${ }^{23}$ This inconsistency may be due to the differences in study population, sample size or age/gender. 

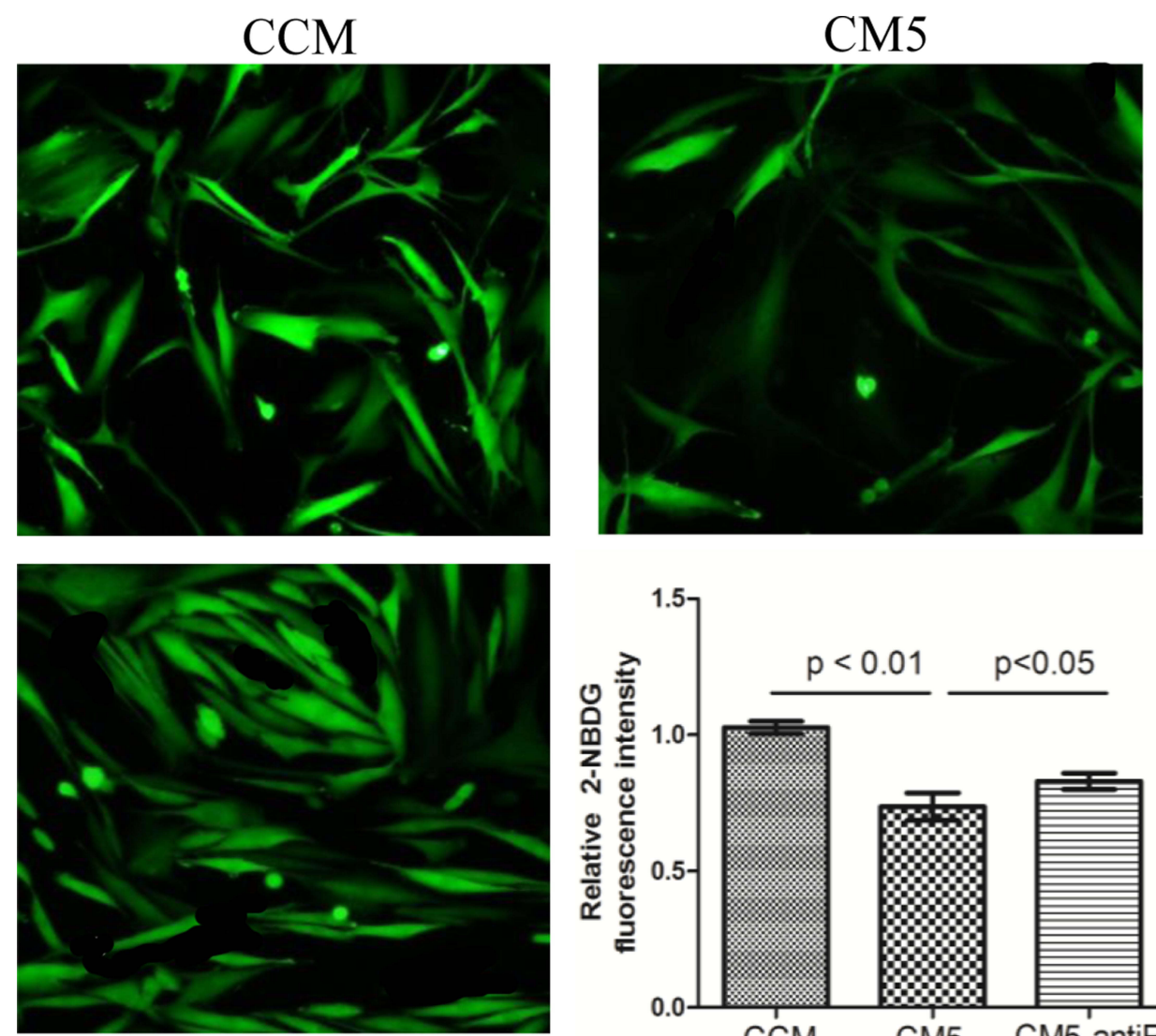

CM5-antiR

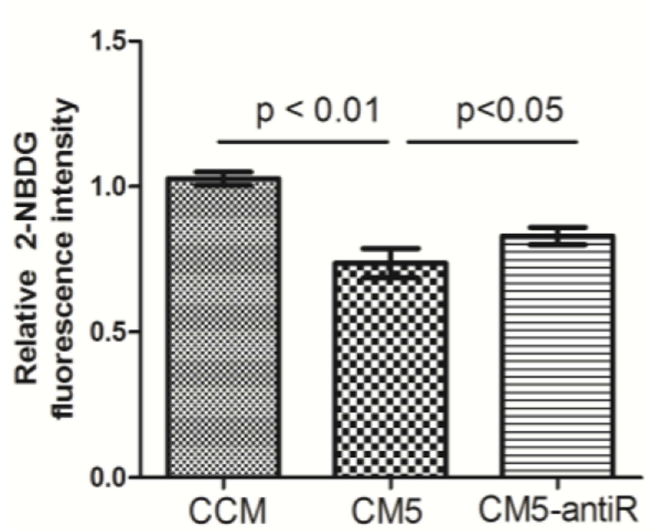

Figure 6 Glucose uptake in $\mathrm{C} 2 \mathrm{Cl} 2$ cell treatment with different conditioned medium (CMM and CM5) and medium mixed with anti-resistin antibody (CM5-antiR). The 2-NBDG fluorescence intensity was significantly increased in $\mathrm{C} 2 \mathrm{Cl} 2$ cells cultured with $\mathrm{CM} 5$-antiR compared with $\mathrm{CM} 5$ group ( $<20.05$ ). CCM: conditioned medium from RAW264.7 without RANKL induced; CM5: conditioned medium from RAW264.7 treated with RANKL for 5 days.

The molecular mechanism of resistin actions is still unclear. Several studies showed that resistin could inhibit insulin receptor substrate-1 and -2 phosphorylation and Akt activation ${ }^{24}$ and can also promote the production of inflammatory cytokine, including tumor necrosis factor $\alpha$ $(\mathrm{TNF}-\alpha)$ and interleukin-6 (IL-6). ${ }^{10}$ Resistin can induce insulin resistance by both AMPK-dependent and AMPKindependent pathways. ${ }^{11}$ Toll-like-receptors (TLR) are considered a major factor in insulin resistance. ${ }^{8,25}$ The receptor or receptors of resistin especially in human is not fully understood. Recent investigations have shown that TLR4 is a potential candidate receptor for human resistin. The resistin/TLR4 signaling pathway may promote the onset of insulin resistance. ${ }^{8}$ Interestingly, TLR4 also plays an important role in $\mathrm{C} 2 \mathrm{C} 12$ myoblasts, such as myogenic differentiation. ${ }^{26,27}$ These data all indicate that resistin may affect $\mathrm{C} 2 \mathrm{C} 12$ cells in glucose uptake-related insulin resistance.

There are several limitations in this study. First, we only performed an in vitro study. Further studies are needed to test the role of osteoclasts on insulin resistance in animal models. Second, a RAW264.7 cell line and primary cell model were adopted in our study. Our results should be confirmed in more ostaoclastic cells model. Third, we did not investigate the mechanism of resistin on insulin resistance because many studies have shown the possible mechanisms. Moreover, the TLR4 pathway has been shown to play important role in the differentiation of $\mathrm{C} 2 \mathrm{C} 12$ cells. Thus, our study could be an exploration.

Our study showed that the resistin expression was increased during osteoclast differentiation, and osteoclasts may affect glucose uptake in $\mathrm{C} 2 \mathrm{C} 12$ cells. Osteoclasts may promote glucose uptake-related insulin resistance by secreting resistin. The inhibition on osteoclast formation may be a potential treatment strategy for insulin resistance or diabetes.

\section{Abbreviations}

$\mathrm{CM}$, conditioned mediums; DMEM, dulbecco's modified Eagle's Medium; ELISA, Enzyme linked immunosorbent 
assay; IR, Insulin resistance; OC, osteocalcin; qRT-PCR, quantitative real-time polymerase chain reaction; TRAP, tartrate resistant acid phosphatase; RANKL, receptor activator of NF-kB ligand.

\section{Data Sharing Statement}

The data used and/or analyzed during the present study are available from the corresponding author on reasonable request.

\section{Acknowledgments}

This work was supported by grants from the Academic Leaders Training Program of Pudong Health Bureau of Shanghai (No. PWRd2019-12), the Talent Project of Shanghai Pudong New Area Gongli Hospital (No. GLRb2017-01), the Postdoctoral Cultivation Fund of Shanghai Pudong New Area Gongli Hospital (No. GLBH 2017005).

\section{Author Contributions}

All authors made a significant contribution to the work reported, whether that is in the conception, study design, execution, acquisition of data, analysis and interpretation, or in all these areas; took part in drafting, revising or critically reviewing the article; gave final approval of the version to be published; have agreed on the journal to which the article has been submitted; and agree to be accountable for all aspects of the work.

\section{Disclosure}

The authors declare that they have no conflicts of interest.

\section{References}

1. DeFronzo RA, Tripathy D. Skeletal muscle insulin resistance is the primary defect in type 2 diabetes. Diabetes Care. 2009;32(Suppl 2): S157-S163. doi:10.2337/dc09-S302

2. Hojlund K. Metabolism and insulin signaling in common metabolic disorders and inherited insulin resistance. Dan Med J. 2014;61(7): B4890.

3. Lee NK, Sowa H, Hinoi E, et al. Endocrine regulation of energy metabolism by the skeleton. Cell. 2007;130(3):456-469. doi:10.1016/ j.cell.2007.05.047

4. Komori T. Functions of osteocalcin in bone, pancreas, testis, and muscle. Int J Mol Sci. 2020;21(20):7513. doi:10.3390/ijms21207513

5. Lasco A, Morabito N, Basile G, et al. Denosumab inhibition of RANKL and insulin resistance in postmenopausal women with osteoporosis. Calcif Tissue Int. 2016;98(2):123-128. doi:10.1007/ s00223-015-0075-5

6. Passeri E, Benedini S, Costa E, et al. A single $60 \mathrm{mg}$ dose of denosumab might improve hepatic insulin sensitivity in postmenopausal nondiabetic severe osteoporotic women. Int $J$ Endocrinol. 2015;2015:352858. doi:10.1155/2015/352858
7. Kiechl S, Wittmann J, Giaccari A, et al. Blockade of receptor activator of nuclear factor- $\mathrm{kB}$ (RANKL) signaling improves hepatic insulin resistance and prevents development of diabetes mellitus. Nat Med. 2013;19(3):358-363. doi:10.1038/nm.3084

8. Benomar Y, Taouis M. Molecular mechanisms underlying obesity-induced hypothalamic inflammation and insulin resistance: pivotal role of resistin/TLR4 pathways. Front Endocrinol. 2019;10:140. doi:10.3389/fendo.2019.00140

9. Shojima N, Sakoda H, Ogihara T, et al. Humoral regulation of resistin expression in 3T3-L1 and mouse adipose cells. Diabetes. 2002;51 (6):1737-1744. doi:10.2337/diabetes.51.6.1737

10. Bokarewa M, Nagaev I, Dahlberg L, et al. Resistin, an adipokine with potent proinflammatory properties. $J$ Immunol. 2005;174 (9):5789-5795. doi:10.4049/jimmunol.174.9.5789

11. Luo Z, Zhang Y, Li F, et al. Resistin induces insulin resistance by both AMPK -dependent and AMPK-independent mechanisms in HepG2 cells. Endocrine. 2009;36(1):60-69. doi:10.1007/s12020009-9198-7

12. Minn AH, Patterson NB, Pack S, et al. Resistin is expressed in pancreatic islets. Biochem Biophys Res Commun. 2003;310 (2):641-645. doi:10.1016/j.bbrc.2003.09.061

13. Patel L, Buckels AC, Kinghorn IJ, et al. Resistin is expressed in human macrophages and directly regulated by PPAR gamma activators. Biochem Biophys Res Commun. 2003;300(2):472-476. doi:10.1016/S0006-291X(02)02841-3

14. Yura S, Sagawa N, Itoh H, et al. Resistin is expressed in the human placenta. J Clin Endocrinol Metab. 2003;88(3):1394-1397. doi:10.1210/jc.2002-011926

15. TD zięgielewska-Gęsiak S, Wyszomirska K, Fatyga E, et al. The role of oxidant-antioxidant markers and resistin in metabolic syndrome elderly individuals. Sci Prog. 2021;104(2):368504211006510.

16. Thommesen L, Stunes AK, Monjo M, et al. Expression and regulation of resistin in osteoblasts and osteoclasts indicate a role in bone metabolism. J Cell Biochem. 2006;99(3):824-834. doi:10.1002/ jcb. 20915

17. Nogueira AVB, Nokhbehsaim M, Tekin S, et al. Resistin is increased in periodontal cells and tissues: in vitro and in vivo studies. Mediators Inflamm. 2020;2020:9817095. doi:10.1155/2020/ 9817095

18. de Paula FJ, Horowitz MC, Rosen CJ. Novel insights into the relationship between diabetes and osteoporosis. Diabetes Metab Res Rev. 2010;26(8):622-630. doi:10.1002/dmrr.1135

19. Bahceci M, Gokalp D, Bahceci S, et al. The correlation between adiposity and adiponectin, tumor necrosis factor alpha, interleukin-6 and high sensitivity C-reactive protein levels. Is adipocyte size associated with inflammation in adults? J Endocrinol Invest. 2007;30 (3):210-214. doi:10.1007/BF03347427

20. Choi SH, Hong ES, Lim S. Clinical implications of adipocytokines and newly emerging metabolic factors with relation to insulin resistance and cardiovascular health. Front Endocrinol. 2013;4:97. doi:10.3389/fendo.2013.00097

21. Hivert MF, Sullivan LM, Fox CS, et al. Associations of adiponectin, resistin, and tumor necrosis factor-alpha with insulin resistance. $J$ Clin Endocrinol Metab. 2008;93(8):3165-3721. doi:10.1210/ jc.2008-0425

22. Norata GD, Ongari M, Garlaschelli K, et al. Plasma resistin levels correlate with determinants of the metabolic syndrome. Eur J Endocrinol. 2007;156(2):279-284. doi:10.1530/eje.1.02338

23. Mostafazadeh M, Haiaty S, Rastqar A, et al. Correlation between resistin level and metabolic syndrome component: a review. Horm Metab Res. 2018;50(7):521-536. doi:10.1055/a-0637-1975

24. Satoh H, Nguyen MA, Miles PD, et al. Adenovirus-mediated chronic "hyper-resistinemia" leads to in vivo insulin resistance in normal rats. J Clin Invest. 2004;114(2):224. doi:10.1172/JCI20785

25. Rogero MM, Calder PC. Obesity, inflammation, toll-like receptor 4 and fatty acids. Nutrients. 2018;10(4):E432. doi:10.3390/nu10040432 
26. Ono Y, Sakamoto K. Lipopolysaccharide inhibits myogenic differentiation of $\mathrm{C} 2 \mathrm{C} 12$ myoblasts through the Toll-like receptor 4-nuclear factor- $\mathrm{\kappa B}$ signaling pathway and myoblast-derived tumor necrosis factor- $\alpha$. PLoS One. 2017;12(7):e0182040. doi:10.1371/journal. pone. 0182040
27. Frost RA, Nystrom GJ, Lang CH. Lipopolysaccharide stimulates nitric oxide synthase-2 expression in murine skeletal muscle and $\mathrm{C}$ (2)C(12) myoblasts via Toll-like receptor-4 and c-Jun NH(2)-terminal kinase pathways. Am J Physiol Cell Physiol. 2004;287(6):C1605-15. doi:10.1152/ajpcell.00010.2004

\section{Publish your work in this journal}

Diabetes, Metabolic Syndrome and Obesity: Targets and Therapy is an international, peer-reviewed open-access journal committed to the rapid publication of the latest laboratory and clinical findings in the fields of diabetes, metabolic syndrome and obesity research. Original research, review, case reports, hypothesis formation, expert opinion and commentaries are all considered for publication. The manuscript management system is completely online and includes a very quick and fair peer-review system, which is all easy to use. Visit http://www.dovepress.com/testimonials.php to read real quotes from published authors. 